\title{
Investigation into the antimicrobial activity of fumarate against Listeria monocytogenes and its mode of action under acidic conditions
}

\section{Article}

Accepted Version

Creative Commons: Attribution-Noncommercial-No Derivative Works 4.0

Barnes, R. H. and Karatzas, K. A. G. (2020) Investigation into the antimicrobial activity of fumarate against Listeria monocytogenes and its mode of action under acidic conditions. International Journal of Food Microbiology, 324. 108614. ISSN 0168-1605 doi:

https://doi.org/10.1016/j.ijfoodmicro.2020.108614 Available at https://centaur.reading.ac.uk/91061/

It is advisable to refer to the publisher's version if you intend to cite from the work. See Guidance on citing.

Published version at: http://dx.doi.org/10.1016/j.ijfoodmicro.2020.108614

To link to this article DOI: http://dx.doi.org/10.1016/j.ijfoodmicro.2020.108614

Publisher: Elsevier

All outputs in CentAUR are protected by Intellectual Property Rights law, including copyright law. Copyright and IPR is retained by the creators or other copyright holders. Terms and conditions for use of this material are defined in the End User Agreement. 


\section{www.reading.ac.uk/centaur}

\section{CentAUR}

Central Archive at the University of Reading

Reading's research outputs online 
1 Investigation into the antimicrobial activity of fumarate against Listeria monocytogenes

2 and its mode of action under acidic conditions

3

4 Ruth H. Barnes ${ }^{\mathrm{a}}$ and Kimon Andreas G. Karatzas ${ }^{\mathrm{a}^{*}}$

5 a Department of Food and Nutritional Sciences, School of Chemistry, Food and Pharmacy,

6 Department of Food \& Nutritional Sciences, University of Reading, Reading RG6 6AD UK.

7

$8 \quad$ Corresponding author.

9

10 Corresponding author address:

11 School of Chemistry, Food and Pharmacy

12 Department of Food \& Nutritional Sciences,

13 University of Reading,

14 Reading

15 RG6 6AD

16 UK

17

18 Tel. +441183786678

$19 \quad$ Fax. +441189310080

20 Email: k.karatzas@ reading.ac.uk

21

22 Keywords: Listeria monocytogenes, fumaric acid, glutamate decarboxylase, inhibition, GAD, 23 biofilm 


\section{Abstract}

28 Organic acids such as fumarate are commonly used as antimicrobials in foods. Apart from the classical mechanism of intracellular dissociation, weak acids are active through important additional mechanisms which are not well-defined. Fumarate, based on its low dissociation constants is expected to have a low antimicrobial activity which is not the case, suggesting additional antimicrobial effects. Previously, fumarate has been shown to inhibit the GAD system of E. coli and therefore, we investigated for first time how it affects this system in Listeria monocytogenes. We found that fumarate is highly antimicrobial towards $L$. monocytogenes under acidic conditions. We also show that in cell lysates and similarly to $E$. coli, fumarate inhibits the GAD system of L. monocytogenes. However, despite the inhibition and in contrast to E. coli, L. monocytogenes is able to counteract this and achieve a higher extracellular GAD output (measured by GABA export) in the presence of fumarate compared to its absence. The latter is achieved by a dramatic 9.44 -fold increase in the transcription of gadD2 which is the main component of the extracellular GAD system. Interestingly, although maleate, the cis-isomer of fumarate results in a more dramatic 48.5-fold gadD2 upregulation than that of fumarate, the final $\mathrm{GAD}_{\mathrm{e}}$ output is lower suggesting that maleate might be a stronger inhibitor of the GAD system. In contrast, the $\mathrm{GAD}_{\mathrm{e}}$ removes more protons in the presence of fumarate than in the presence of $\mathrm{HCl}$ at the same $\mathrm{pH}$. All the above suggest that there are additional effects by fumarate which might be associated with the intracellular GAD system $\left(\mathrm{GAD}_{\mathrm{i}}\right)$ or other acid resistance systems. We assessed the $\mathrm{GAD}_{\mathrm{i}}$ output by looking at the intracellular GABA pools which were not affected by fumarate. However, there are multiple pathways (e.g. GABA shunt) that can affect $\mathrm{GABA}_{i}$ pools and we can not conclusively suggest that $\mathrm{GAD}_{\mathrm{i}}$ is affected. Furthermore, similarly to maleate, fumarate is able to eliminate $L$. monocytogenes in biofilms under acidic conditions. Overall, fumarate is a good candidate for 
51 L. monocytogenes decontamination and biofilm removal which is not toxic compared to the

52 toxic maleate.

\section{1. Introduction}

55 Foodborne illness is a significant public health problem both in the UK and globally. The 56 World Health Organisation (WHO) estimates that foodborne illness is responsible for 2.2 57 million deaths annually (Food standards agency, 2011). The majority of this illness in the UK 58 is caused by Campylobacter and Norovirus whilst most deaths are due to Listeria 59 monocytogenes and Escherichia coli infections. (Food standards agency, 2011)

60 Various strategies are employed to eliminate these pathogens in foods aiming to reduce the 61 incidence of foodborne illness. One such strategy is the addition of organic acids which have 62 been used for centuries to prevent the growth of pathogenic and spoilage bacteria (Ricke, 2003).

63 Organic acids are believed to affect microorganisms through diffusion of undissociated molecules across the cell membrane followed by intracellular dissociation and release of 65 protons causing death or growth inhibition (Comes and Beelman, 2002; Lambert and Stratford, 66 1999; Podolak et al., 1996).

67 One of the well-known organic acids with antimicrobial activity is fumaric acid, which is a 68 food grade, dicarboxylic acid found widely in nature and active against a number of foodborne 69 pathogens including E. coli, L. monocytogenes and Salmonella sp. (Comes and Beelman, 2002; 70 Kim et al., 2009; Kondo et al., 2006; Miller and Kaspar, 1994; Pérez - Díaz and McFeeters, 71 2010; Podolak et al., 1996). In the EU and the US besides as an antimicrobial is also used as an 72 acidulant, and a flavour enhancer (Lee, 2014; Saltmarsh et al., 2013). Fumaric acid is regularly 73 used in various products including baked goods, confectionery, juices and dried powdered 74 foods as well as in animal feed ( Lee, 2014). Fumaric acid is considered as one of the relatively strongest among the weak organic acids, which however has low solubility in aqueous solutions 
76 (Arnold et al., 2001; Roa Engel et al., 2013) while its salts are highly soluble (Zhou et al.,

77 2002). Based on its low dissociation constants ( $\mathrm{p} K_{\mathrm{a} 1}=3.02$ and $\mathrm{p} K_{\mathrm{a} 2}=4.38$; Lohbeck et al.,

78 2000; Okuyama and Maskill, 2013; Szalka et al., 2013) it should be expected that fumarate has

79 low antimicrobial activity although this is not the case, if compared to other organic acids

80 including acetic and lactic acid (Podolak et al., 1996). This additional antimicrobial activity of

81 fumarate, beyond what could be explained by the intracellular dissociation theory of weak

82 acids, is normally attributed to unknown factors such as interference with metabolic activities

83 stress mechanisms or other cellular functions. Understanding these additional effects could

84 increase our knowledge and allow us to enhance the antimicrobial activity of these compounds

85 and consequently achieve higher levels of hygiene or develop novel and improved

86 antimicrobial regimes. Furthermore, it is important to understand in detail against which

87 organisms this additional effect occurs and what the mode of action is.

88 The current study focuses on the foodborne pathogen L. monocytogenes, a Gram positive,

89 facultative anaerobic bacterium that is ubiquitous in the environment (Posfay-Barbe and Wald,

90 2009) causing listeriosis, that mainly affects pregnant women, neonates and

91 immunocompromised individuals (Posfay-Barbe and Wald, 2009). The organism is capable of

92 surviving a wide range of environmental conditions and can grow under refrigeration

93 temperatures affecting ready-to-eat products (Liu et al., 2002; O'Driscoll et al., 1996). It is also

94 able to survive extreme acidic environments such as the stomach or acidic foods through the

95 use key mechanisms of acid resistance of which the main one is the GAD system (Davis et al.,

96 1996; Foster, 2004).

97 The GAD system converts glutamate to $\gamma$-amino butyric acid (GABA) with the removal of a

98 proton resulting in an increase in the intracellular pH (Cotter et al., 2001; Karatzas et al., 2012).

99 The architecture of the GAD system is highly variable and in L. monocytogenes it typically

100 comprises two antiporters, GadT1 and GadT2 and three decarboxylases GadD1, GadD2 and 
GadD3. The GadD1T1 operon is typically associated with growth under mild acidic conditions, the GadT2D2 promoting survival under extreme acidic conditions (Cotter et al., 2005) while the GadD3 is the main part of the intracellular GAD system $\left(G_{A} D_{i}\right)$ utilising solely intracellular glutamate to produce intracellular GABA which is catabolised to glutamate by the GABA shunt (Cotter and Hill, 2003; Feehily et al., 2014; Feehily and Karatzas, 2013).

Given that fumarate has previously been described as an inhibitor of the E. coli GAD enzyme (Fonda, 1972) we investigate here the antimicrobial activity of fumarate on L. monocytogenes under acidic conditions and the possibility that this stems from effects on the GAD system and possibly other aminoacid decarboxylase systems (Grobelny, 1995). Furthermore, we look at the ability of fumarate to remove biofilms of L. monocytogenes and investigate further the effects of the cis-isomer of fumarate, maleic acid on the GAD system which has also been previously shown to affect it in L. monocytogenes (Paudyal et al., 2018).

\section{2. Materials and methods}

\section{$115 \quad 2.1$ Bacterial strains and growth conditions}

116 All strains (Table 1) were stored in $2 \mathrm{ml}$ cryovials with a 7\% dimethyl sulfoxide (DMSO) at $80^{\circ}$ C. L. monocytogenes $10403 \mathrm{~S}$ and EGD-e were cultured onto Brain Heart Infusion (BHI) agar (LABM, Lancashire UK) and L. monocytogenes LO28 onto Tryptic Soy Broth (Oxoid, UK) supplemented with 5\% yeast extract (TSBY; Oxoid, UK) and incubated at $37^{\circ} \mathrm{C}$ overnight. Three colonies from each plate were transferred, with an inoculation loop, into BHI and TSBY broth respectively in $10 \mathrm{ml}$ Sterilin polystyrene tubes and incubated at $37^{\circ} \mathrm{C}$ with shaking $(150$ rpm) for $18 \mathrm{~h}$. These overnight cultures were used to inoculate $20 \mathrm{ml}$ cultures of the corresponding media (1\% inoculum) in $250 \mathrm{ml}$ conical flasks which then were subsequently incubated overnight at $37^{\circ} \mathrm{C}$ with shaking at $(150 \mathrm{rpm})$ for $18 \mathrm{~h}$. 
127 The percentage of undissociated acid present at pH 3 was determined using the HendersonHasselbalch equation as adapted by Wemmenhove et al., (2016) and presented at Table 2 $[$ Undissociated acid $]=[$ Total acid $] / 1+10^{(\mathrm{pH}-\mathrm{pKa})}$

\subsection{Determination of minimum inhibitory concentration}

A range of concentrations $(0,1,2,4,8,16$ and $32 \mathrm{mg} / \mathrm{ml}$ ) of selected organic acids (Table 3 ) were used in BHI inoculated at $1 \%$ with overnight culture of L. monocytogenes $10403 \mathrm{~S}$ WT. Cultures were then placed into 96 well plates and had their $\mathrm{OD}_{620 \mathrm{~nm}}$ analysed over a $24 \mathrm{~h}$ period using a Sunrise plate reader machine (Tecan, Mannedorf, Switzerland) operated by Magellan software (Tecan, Mannedorf, Switzerland) with 20 min intervals between measurements. The plates were kept at $37^{\circ} \mathrm{C}$ with shaking to assess the MIC.

\subsection{Survival under acidic conditions}

Survival experiments were undertaken with L. monocytogenes 10403S WT. Twenty ml cultures were prepared in BHI, using stock cultures prepared as described previously, and grown in $250 \mathrm{ml}$ Erlenmeyer flasks at $37^{\circ} \mathrm{C}$ with agitation at $150 \mathrm{rpm}$. Acid challenge took place with the addition of $8.6 \mathrm{mM}$ fumaric acid, and a variety of organic acids (Table 3 ). Control experiments were performed in the absence of any chemicals in overnight cultures. The $\mathrm{pH}$ of the cultures was then adjusted to $\mathrm{pH} 3.0 \mathrm{using} 1 \mathrm{M} \mathrm{HCl}$.

One hundred $\mu \mathrm{l}$ samples were obtained prior to the acid challenge and every $20 \mathrm{~min}$ for $1 \mathrm{~h}$ and placed in $900 \mu \mathrm{l}$ Maximum Recovery Diluent (MRD; Oxoid Limited, Hampshire UK). Tenfold serial dilutions were prepared and $10 \mu \mathrm{l}$ of each dilution were placed onto BHI agar plates using the spot plate method and incubated at $37^{\circ} \mathrm{C}$ overnight. Subsequently, colonies were 
counted to assess the cell concentration in the culture at each time point. All experiments were performed in triplicate.

\section{$153 \quad 2.5$ Survival in the presence of sodium fumarate}

154 Following initial survival experiments, further survival experiments were performed focusing on the effect of fumaric acid and its salt sodium fumarate on L. monocytogenes 10403S WT, and its isogenic mutants $\Delta g a d D 1, \Delta g a d D 2, \Delta g a d D 3$, on EGD-e WT and its isogenic mutants $\Delta g a d D 1, \Delta g a d D 2, \Delta g a d D 3$ and on LO28 WT with its isogenic mutants $\Delta g a d D 1, \Delta g a d D 2$,

$158 \Delta g a d D 1 / 2$.

159 Cultures were prepared in BHI or TSBY for LO28, using stock cultures, prepared as described previously and grown in $250 \mathrm{ml}$ Erlenmeyer flasks at $37^{\circ} \mathrm{C}$ with agitation at $150 \mathrm{rpm}$. Due to the significantly differences in strain sensitivity, different concentrations of sodium fumarate were used for 10403S (8.6 mM) and for EGD-e and LO28 (4.3 mM). Control cultures were also prepared containing no additional antimicrobials. Subsequently, all L. monocytogenes 164 10403S cultures had their $\mathrm{pH}$ adjusted to 3.0 and L. monocytogenes EGD-e and LO28 to $\mathrm{pH}$ 1653.3.

166 One hundred $\mu \mathrm{l}$ samples were taken immediately prior to the acid challenge and every 20 or 5 167 min thereafter for 10403 S or EGD-e and LO28 respectively. Samples were subsequently added 168 in $900 \mu \mathrm{l}$ MRD (Oxoid Limited, Hampshire UK) to prepare decimal serial dilutions and $10 \mu \mathrm{l}$ 169 of each dilution was plated onto BHI agar or TSBY agar respectively and incubated at $37^{\circ} \mathrm{C}$ overnight. Following incubation, colonies were counted to assess the cell concentration at every 171 time point. 
174 GABase assay was used to determine the concentrations of intracellular $\left(\mathrm{GABA}_{\mathrm{i}}\right)$ and

175

176

177 extracellular (GABA $)$ GABA in $10403 \mathrm{~S}$ and LO28 WT as described by O'Byrne et al., (2011). E. coli $\mathrm{K}-12$ samples were assayed following the same methodology with the modification of the initial culture being grown in Lysogeny broth (LB LAB M, Lancashire, UK) supplemented with $10 \mathrm{mM}$ monosodium glutamate (MSG; Steinheim, Germany), GABAe was quantified according to Tsukatani et al. (2005) as modified by Karatzas (2010).

\subsection{GAD activity in protein lysates}

WT $10403 \mathrm{~S}$ cultures were prepared in $20 \mathrm{ml} \mathrm{BHI} \mathrm{in} 250 \mathrm{ml}$ Erlenmeyer as described previously by Paudyal et al (2018), while for LO28 and EGD-e $40 \mathrm{ml}$ cultures were used. All cultures were then transferred to $50 \mathrm{ml}$ falcon tubes (VWR, Leighton Buzzard UK) with $10 \mu \mathrm{g} / \mathrm{ml}$ chloramphenicol (Sigma-Aldrich, Steinheim, Germany). The samples were then centrifuged at $12,000 \mathrm{x}$ g for $15 \mathrm{~min}$ and washed with a buffer solution, as described previously (Abrams et al., 2008; Boura et al., 2016). Suspensions were then incubated at $37^{\circ} \mathrm{C}$ in an orbital shaker at 150 rpm (Gallenkamp, Germany).

A $2 \mathrm{ml}$ cryovial (Sarstedt, Germany) was filled with $0.07 \mathrm{~g}$ acid washed glass beads $(<106 \mu \mathrm{m}$ diameter Sigma-Aldrich, Steinheim, Germany) together with $1 \mathrm{ml}$ cell suspension. The sample was then agitated using a Mini-Beadbeater (Biospec, Bartesville, USA), thrice for 1 min, followed by 1 min on ice. DNAse I (Thermo Fisher Scientific, California, USA) was then added up to $0.1 \%$ in the cell lysates and were then incubated at $37^{\circ} \mathrm{C}$ in an orbital shaker at $150 \mathrm{rpm}$ for $30 \mathrm{~min}$. One $\mathrm{ml}$ sample was then transferred to an Eppendorff tube and centrifuged at 5,000 $\mathrm{x} g$ for $15 \mathrm{~min}$. The supernatant was then transferred to an Eppendorf tube and the pellet discarded. Subsequently, $100 \mu$ of the supernatant was added to a pyridine hydrochloride buffer (Fonda, 1972) supplemented with $30 \mathrm{mM}$ MSG and with or without $20 \mathrm{mM}$ sodium fumarate. All samples were then adjusted to a $\mathrm{pH}$ of 4.5. The GABase assay was then used to assess 
GABA levels. It had previously been established using standard concentrations of GABA, that the presence of sodium fumarate does not affect the accuracy of this assay.

201

\subsection{Amino acid analysis by GC-MS}

203 As the activity of the GABase enzyme could be affected by the presence of other molecules, 204 GABA concentrations were also assessed in the supernatant or the bacterial lysates with the use of gas chromatography mass spectrometry (Elmore et al., 2005). The method also assessed the concentration of a wide range of aminoacids. Intra- and extra-cellular samples taken from L. monocytogenes $10403 \mathrm{~S}$ were assessed in the presence and absence of sodium fumarate. Previous work by Paudyal et al. 2018 has shown that in similar conditions to those described here, GABA levels quantified by GC-MS and GABase were always within $\pm 5 \%$.

\subsection{Real-time PCR determination of GAD gene expression}

212 The transcription of the gad genes in the presence and absence of sodium fumarate was assessed 213 in L. monocytogenes 10403S WT (gadD1, gadD2 and gadD3) using real time reverse 214 transcription-PCR (RT-PCR) as previously described by Karatzas et al., (2010). The transcription of the antiporter-encoding genes ( $\operatorname{gadT1}$ and $\operatorname{gadT2})$ was not examined as it has previously been demonstrated that it is similar to the corresponding glutamate decarboxylases (gadD1 and gadD2) belonging to the same corresponding operon (Karatzas et al., 2012).

218 Overnight cultures of L. monocytogenes 10403S WT grown for $24 \mathrm{~h}$ until stationary phase in 219 BHI were treated with $10 \mathrm{mM}$ of either sodium fumarate or maleic acid for $40 \mathrm{~min}$. Samples were taken and prepared as previously described by Karatzas et al (2010). Relative expression of the data was calculated as a ratio between expression of each of the target genes and the expression of the 16S rRNA which was used as the reference gene for each cDNA sample. 
Light Cycler 480 SW 1.5.1 software programme, with PCR efficiencies of the primer pairs gadD1F-gadD1R, gadD2F-gadD2R, gadD3F-gadD3R and 16SF-16SR being 2.12, 2.09, 2.03 and 2.27 respectively (Karatzas et al., 2010).

\subsection{Biofilm removal by sodium fumarate}

Biofilm formation was assessed using L. monocytogenes 10403S WT, EGD-e WT and LO28 WT overnight cultures grown in their corresponding BHI or TSBY agar as described previously and then inoculated to $1 \%$ in a $2 \mathrm{ml}$ of BHI broth or TSBY broth. The broth was mixed thoroughly and placed in a 24-flat-bottom-well Corning Costar cell culture plate and sealed using petrifilm. Following incubation at $37^{\circ} \mathrm{C}$ for $48 \mathrm{~h}$, the culture was removed and the wells washed thrice with sterile water. Subsequently a fourth treatment was applied using $2.5 \mathrm{ml}$ of either water, $100 \mathrm{ppm}$ free chlorine from calcium hyperchlorite, $\mathrm{HCl}(\mathrm{pH} 2.4), \mathrm{HCl}(\mathrm{pH} 2.4)$ with $25 \mathrm{mM}$ sodium fumarate, AM (an organic acid disinfectant) at $\mathrm{pH} 2.4$ and $\mathrm{AM}$ at $\mathrm{pH} 2.4$ with $25 \mathrm{mM}$ of sodium fumarate. The biofilm was exposed to these solutions in the well for 5 min and then the supernatants were discarded and wells were rinsed with $2.5 \mathrm{ml}$ deionized water. Subsequently, $500 \mu 1$ MRD was placed in the well and the bottom of the well was scraped using a $200 \mu \mathrm{l}$ pipette tip for $30 \mathrm{~s}$ in a pattern covering the whole well bottom. This was repeated 4 times to provide a total volume of $2 \mathrm{ml}$ which was serially diluted 10 -fold and then $10 \mu \mathrm{l}$ was plated onto BHI or TSBY agar and incubated at $37^{\circ} \mathrm{C}$ for $24 \mathrm{~h}$ and then growth was assessed (Ramírez et al., 2015). The impact of the treatments on the biofilm was assessed using the following calculation (Hamilton, 2003; Heersink J., 2003; Ramírez et al., 2015).

Density $=($ Average count $/$ Volume plated $) *$ Dilution $*$ Volume of MRD scraped into $*(1 /$ well surface area).

\subsection{Statistical analysis}


In all cases all experiments were run in triplicate unless stated otherwise. Subsequently results were assessed using paired Student t-tests. A P value below 0.05 indicated a statistically significant result accompanied by an asterisk.

$253 \quad 3$ Results

254 3.1 Calculation of the percentage of undissociated acid

255 The pKa of sodium fumarate is low compared to the other acids tested (maleic acid, tartaric 256 acid and oxaloacetic acid; Table 2). This suggest that fumaric acid has a lower level of 257 undissociated acid $(51.7 \%)$ and therefore, lower antimicrobial activity.

\subsection{Growth in the presence of selected acids}

The MICs of a variety of organic acids on L. monocytogenes 10403S WT were assessed (Table

3). However, tartaric acid seemed to be the most bacteriostatic as it had the lowest MIC (14.9 $\mathrm{mM})$. Sodium fumarate as a salt did not inhibit L. monocytogenes under the conditions of the current experiment.

\subsection{Acid survival of L. monocytogenes 10403S, LO28 and EGD-e in the presence of} different organic acids.

Under acidic conditions ( $\mathrm{pH}$ 3), $8.6 \mathrm{mM}$ sodium fumarate showed a significant bactericidal effect on survival of the 10403S (Fig. 1A). Similar results were obtained with both EGD-e and LO28 (Fig. 1B and 1C) assessed at pH 3.3 as more acid-sensitive than 10403S (Karatzas et al., 2012). Based on previous work, it was expected that EGD-e might be the most sensitive strain, however it displayed a similar response with LO28 to sodium fumarate (Fig. 1B and 1C;

272 Karatzas et al., 2012). Furthermore, we tested survival of 10403S against all compounds 273 mentioned in Table 1, and fumaric acid, sodium fumarate and maleic acid were the most 
bactericidal at $8.6 \mathrm{mM}$ and $\mathrm{pH} 3.3$ with all other compounds hardly conferring any inactivation

275 (data not shown).

\subsection{Survival of L. monocytogenes 10403S LO28 and EGD-e and their isogenic mutants} under acidic conditions in the presence and absence of sodium fumarate.

279 Once it was determined that sodium fumarate conferred the highest bactericidal activity, the role of the GAD genes in the presence of sodium fumarate was assessed. In all cases, the presence of sodium fumarate resulted in significant increase in the log reduction in all WT strains and mutants (Fig. 2).

In $10403 \mathrm{~S}$ the absence of sodium fumarate at $\mathrm{pH} 3$ with $\mathrm{HCl}$, minor log reductions in survival occurred with $\Delta$ gadD2 being the most sensitive. In the presence of $8.6 \mathrm{mM}$ sodium fumarate (pH 3) a significantly higher log reduction occurred for all strains while a similar trend occurred, with all mutants except $\Delta$ gadD2, behaving similarly to the WT (10403S WT, $\Delta$ gadD1 and $\Delta$ gadD3 showed 2.29, 1.99, and $2.56 \log$ reduction of CFU/ml respectively; Fig. 2A). $\Delta g a d D 2$ was the most sensitive strain and impacted more by the presence of sodium fumarate (5.21 log reduction of $\mathrm{CFU} / \mathrm{ml})$.

In LO28 the effect of sodium fumarate showed a similar trend to 10403S, although this strain was more sensitive and the effect was significantly more pronounced. Also, in this case the addition of $4.3 \mathrm{mM}$ sodium fumarate significantly affected survival at $\mathrm{pH} 3.3$ and gadD2 was also in this case the main determinant either with $\mathrm{HCl}$ alone or with sodium fumarate (Fig. 2B).

294 In the presence of $4.3 \mathrm{mM}$ sodium fumarate (pH 3.3), LO28 WT and $\Delta$ gadD1, showed a 4.80 and $5.11 \log$ reduction $\mathrm{CFU} / \mathrm{ml}$ respectively while that of $\Delta g a d D 2$ and $\Delta g a d D 1 / 2$ was higher than the maximum of $6 \operatorname{logs}$ that could be determined with this protocol (Fig. 2B). In EGD-e the major difference compared to the other two strains was that removal of gadD2 did not result in increased sensitivity. In the presence of $4.3 \mathrm{mM}$ sodium fumarate $(\mathrm{pH} 3.3$ ), 

probably due to the activation of another acid resistance mechanism. This trend had also been observed in the presence of maleic acid the $\mathrm{GAD}_{\mathrm{i}}$ system may play a survival role in the presence of sodium fumarate (Paudyal et al., 2018).

\subsection{Extracellular GABA of L. monocytogenes 10403S, LO28 and E. coli K-12.}

To assess the possible influence of sodium fumarate on the activity of the GAD system an examination of the effect of this compound on the levels of $\mathrm{GABA}_{\mathrm{e}}$ was undertaken while the cells were in stationary phase. In L. monocytogenes 10403S WT the presence of sodium fumarate resulted in significant higher $\mathrm{GABA}_{\mathrm{e}}$ levels from (4.11 mM GABA) compared to its absence at pH 4.2 (2.01 mM GABA; 2.04-fold higher GABA levels $\mathrm{P}<0.05$; paired T-test, Fig. 3A). Similarly, higher levels were obtained with all isogenic mutants except $\Delta$ gadD2 (data not shown). Similarly, LO28 WT also showed higher GABA levels in the presence of sodium fumarate (2.89 $\mathrm{mM} \mathrm{GABA}_{\mathrm{e}}$ ) compared to $1.24 \mathrm{mM} \mathrm{GABA}_{\mathrm{e}}$ in its absence (2.33-fold higher GABA $_{e}$ levels Fig. 3B) although this result was not statistically significant. Also, its isogenic mutants followed the same pattern (data not shown). These experiments were not performed with EGD-e as it is defective in extracellular GABA production.

318 Finally, when a similar experiment was undertaken using E. coli $\mathrm{K}-12 \mathrm{WT}(\mathrm{pH}$ 4) in contrast 319 to the above, $\mathrm{GABA}_{\mathrm{e}}$ levels were significantly lower $(4.5 \mathrm{mM} \mathrm{GABA})$ in the presence of sodium fumarate compared to its absence $\left(9.2 \mathrm{mM} \mathrm{GABA}_{\mathrm{e}} ; 2.01\right.$-fold lower levels, $\mathrm{P}<0.05$; paired T-test; Fig. 3C). GABA $\mathrm{i}_{\mathrm{i}}$ was also examined however, no significant difference in any of the strains tested was observed in the presence of sodium fumarate. 


\subsection{S WT GAD activity in protein lysates}

325 The GAD activity of protein lysates was assessed by monitoring GABA production in the 326 presence of MSG (Sigma-Aldrich, Steinheim, Germany). The results indicate that, sodium 327 fumarate inhibited the GAD system activity in 10403S WT, resulting in reduced levels of 328 GABA from $3.4 \mathrm{mM}$ to $2.7 \mathrm{mM}$ (0.79-fold reduced levels; P <0.05; Fig. 4). A similar protocol was attempted with EGD-e and LO28 however, GABA levels were below the detection limit of the GABase assay and despite protocol alterations in the $\mathrm{pH}$, the buffer used, higher glutamate supplementation or increasing the volume of culture utilised no improvement occurred (Fig. 5).

\subsection{Real-time PCR determination of GAD gene expression.}

335 Real time quantitative Polymerase Chain Reaction (RT-qPCR) was used to quantify the transcription of the L. monocytogenes 10403S WT GAD system genes in the presence of sodium fumarate and its cis-isomer maleic which has previously been shown to inhibit the listerial GAD system (Paudyal et al., 2018). Transcription of gadDl was very low and not affected by the presence of sodium fumarate or maleic acid (Fig. 6A). In contrast, both sodium fumarate and maleic acid resulted in a significant upregulation $(\mathrm{P}<0.05)$ of the main component of the $\mathrm{GAD}_{\mathrm{e}}$ system, gadD2 by 9.44- and 48.51-fold respectively (Fig. 6B). The latter gene also showed the highest expression compared to the other two decarboxylases. Regarding gadD3, expression was not affected by the presence of sodium fumarate although that of maleic acid showed to result in an increase of 22.33 -fold which however, was not statistically significant 345 (Fig. 6C). 
348 The survival of L. monocytogenes biofilms was assessed after the application of various 349 antimicrobial treatments including $100 \mathrm{ppm}$ chlorine and an acidic disinfectant (AM).

350 When L. monocytogenes $10403 \mathrm{~S}$ was assessed, all treatments did not affect the survival in the 351 biofilm with the exception of those with $25 \mathrm{mM}$ sodium fumarate, either alone at $\mathrm{pH} 2.4(1 \mathrm{M}$ $352 \mathrm{HCl} ; 1.49 \log$ reduction of $\left.\mathrm{CFU} / \mathrm{cm}^{2}\right)$ or in combination with $\mathrm{AM}$ at the same $\mathrm{pH}(1.98 \log$ 353 reduction of $\mathrm{CFU} / \mathrm{cm}^{2}$; Fig. 7A).

354 In L. monocytogenes EGD-e three treatments achieved a statistically significant reduction of $355 \mathrm{CFU} / \mathrm{ml}$. Those were the same ones that affected 10403S and the AM disinfectant alone (2.35 $356 \log$ reduction of $\mathrm{CFU} / \mathrm{cm}^{2}$; Fig. 7B). Sodium fumarate alone at $\mathrm{pH} 2.4$ resulted in a significant $3573.72 \mathrm{log}$ reduction of $\mathrm{CFU} / \mathrm{cm}^{2}$ while in combination with $\mathrm{AM}$ resulted in a $4.7 \mathrm{log}$ reduction 358 of $\mathrm{CFU} / \mathrm{cm}^{2}$.

359 In L. monocytogenes LO28 all treatments resulted in a significant reduction in biofilm survival. 360 However, this was due to a lower variability between the replicates and overall the results were 361 similar to EGD-e with the exception of the AM treatment which seemed to be highly effective 362 against this strain. In this case also the two treatments with sodium fumarate were the most 363 effective along with $100 \mathrm{ppm}$ chlorine (2.96 log reduction of CFU/ $\left.\mathrm{cm}^{2}\right)$. Sodium fumarate alone 364 at $\mathrm{pH} 2.4$ resulted in a significant $2.67 \mathrm{log}$ reduction of $\mathrm{CFU} / \mathrm{cm}^{2}$ while in combination with $365 \mathrm{AM}$ resulted in a $3.40 \mathrm{log}$ reduction of $\mathrm{CFU} / \mathrm{cm}^{2}$. The $\mathrm{AM}$ disinfectant treatment at $\mathrm{pH} 2.4$ 366 resulted in a $2.23 \mathrm{log}$ reduction of $\mathrm{CFU} / \mathrm{cm}^{2}$ while $\mathrm{pH} 2.4$ alone resulted in $1.13 \mathrm{log}$ reduction 367 of $\mathrm{CFU} / \mathrm{cm}^{2}$ (Fig. 7C).

\section{Discussion}

370 The antimicrobial effects of organic acids are mainly explained by the theory of passive 371 diffusion of undissociated molecules and their intracellular dissociation (Foster, 2004) which 372 also dictates that organic acids that dissociate more (higher $\mathrm{Ka}$ ) are less antimicrobial, while 
those that dissociate less (lower Ka) more antimicrobial. However, there are major deviations to this rule (Ricke, 2003) suggesting the existence of additional effects which are highly important for our understanding of the mode of action of various organic acids and the behaviour of microorganisms in foods and many other environments.

The present work focuses on fumaric acid which is widely used in foods while it is present in all foods, all living organisms and various environments as it is a key component of the TCA cycle. It is one of the relatively strongest among the weak organic acids and based on its low pKa of 3.02 it should have low antimicrobial activity as it dissociates more (Table 2). Therefore, as predicted, the MIC of fumaric acid was among the highest (34 $\mathrm{mM}$ ) with only oxaloacetic acid $(60.5 \mathrm{mM})$ and sodium fumarate having higher MICs (Table 3).

However, in contrast to the above fumarate showed high bactericidal activity against all three strains of L. monocytogenes used (Fig. 1A, B \& C). This is a clear deviation from the behaviour that would be predicted by the intracellular dissociation theory of weak acids suggesting additional antimicrobial effects which however, only occur at highly acidic conditions. This high antimicrobial activity of fumarate has been noted previously (Chikthimmah et al., 2003; Comes and Beelman, 2002; Podolak et al., 1996; Kondo et al., 2006) and our aim was to identify the complementary mode of action of fumarate which does not stem from the theory of intracellularly dissociation of organic acids.

Fumarate is an inhibitor of the E. coli GAD system (Fonda, 1972) and based on this, an obvious hypothesis to explain the above effects, is the inhibition of the L. monocytogenes GAD system. To investigate this hypothesis and in parallel identify which component of the GAD system might be affected, deletion mutants in GAD decarboxylase genes for all three strains of $L$. monocytogenes were used. In 10403S, removal of gadD1 and gadD3 resulted in similar population reduction when compared to the WT in presence of sodium fumarate. However, removal of gadD2 caused the greatest death both in the presence and in the absence of sodium 
fumarate as expected since most strains rely on the GadD2 for the operation of the dominant $\mathrm{GAD}_{\mathrm{e}}$ system (Fig. 2A; Karatzas, Brennan et al. 2010). Similarly, to 10403S, in LO28 the removal of the key gadD2 significantly reduced survival under acidic conditions (Fig. 2B). However, EGD-e does not possess a GAD e system associated with GadD2, and only utilises the $\mathrm{GAD}_{\mathrm{i}}$ which is mediated by GadD3 (Feehily et al., 2014; Karatzas et al., 2012). In this strain, $\Delta$ gadD3 was the most sensitive either in the presence or absence of sodium fumarate, without statistical significance though, whereas gadD1 and gadD2 removal significantly increased resistance but only in the presence of sodium fumarate (Fig. 2C). Overall inactivation was much higher in the presence of sodium fumarate and more exaggerated for the more sensitive mutants that showed higher inactivation in the absence of sodium fumarate.

Furthermore, it should be noted that removal of gadDl from all strains did not significantly influence their ability to survive acidic conditions. This gene has previously been linked to increased growth under mild acidic conditions based on observations with WT strains that do not possess it (e.g. serotype $4 \mathrm{~b}$ strains; Cotter et al., 2005). However, mutants in this operon 412 have never been shown to possess an acid sensitive phenotype, or evidence showing GadD1T1 exporting GABA neither in the literature or in our experiments, raising questions over its function as a glutamate decarboxylase. Overall, GadD2 and GadD3, depending on the strain, were significant for survival against sodium fumarate under acid conditions (Fig. 2A, B \& C). Sodium fumarate resulted in significant increase in $\mathrm{GABA}_{\mathrm{e}}$ exported by L. monocytogenes 417 10403S (2.04-fold increase) at pH 4.2 (P <0.05; Fig. 3A). Interestingly, this increased $\mathrm{GAD}_{\mathrm{e}}$ output did not confer increased survival under acidic conditions (Fig. 2A). This is highly interesting as each GABA molecule exported, removes one intracellular proton and based on the above, $\mathrm{GAD}_{\mathrm{e}}$ removes twice more protons in the presence than in the absence of fumarate,

421 but instead of conferring higher resistance the opposite occurs (Fig. 2A \& B). A similar trend 422 was observed with LO28 although it was not statistically significant (P >0.05; Fig. 3B). It 
423 should be stated that as we have shown previously, maleic acid, the cis-isomer of fumaric acid

424 works in an opposite fashion than fumarate, as it reduces $\mathrm{GAD}_{\mathrm{e}}$ output (Paudyal et al., 2018).

425 However, in contrast to L. monocytogenes, when we challenged E. coli K12, sodium fumarate 426 resulted in a significant -2.01 -fold decrease in $\mathrm{GABA}_{\mathrm{e}}$ export $(\mathrm{P}<0.05$; Fig. 3C). The latter was 427 expected as fumarate is an inhibitor of E. coli GAD enzyme (Fonda, 1972) and it demonstrates 428 a different GAD system behaviour between these two organisms. Further work should investigate if this is a different feature between Gram-positive and Gram-negative bacteria.

To further assess the effect of sodium fumarate on the GAD system, we measured GABA

431 levels, and found no significant effect of sodium fumarate. At first glance, this might suggest 432 no effect of sodium fumarate however, GABA $\mathrm{A}_{\mathrm{i}}$ levels are also affected by its metabolic flux through the GABA shunt pathway and therefore the above results are not conclusive.

434 Surprisingly, we observed that sodium fumarate significantly inhibited GAD activity as measured through GABA levels in L. monocytogenes $10403 \mathrm{~S}$ cell lysates ( $\mathrm{P}<0.05$; Fig. 4). This coincides with its role as GAD inhibitor in E. coli (Fonda, 1972) and in plants (Ohno and

437 Okunuki, 1962). We further investigated these inhibitory effects of sodium fumarate in lysates of LO28 and EGD-e but unfortunately, we were not able to get measurable GABA levels (Fig. 5) even in the absence of sodium fumarate, despite various protocol modifications (usage of higher cell numbers, higher levels of glutamate, different buffer $\mathrm{pH}$ values). This might be related to lower GAD activity or a different optimal $\mathrm{pH}$ of the GAD enzymes in these strains.

442 Further we looked at the effect of fumarate on transcription of GAD genes gadD1, gadD2 and gadD3 including maleic acid which is a cis-isomer of fumarate and we have previously shown that reduces GAD output and activity in L. monocytogenes enhancing its acid sensitivity (Paudyal et al., 2018). RT-qPCR showed no effect of fumarate or maleate on gadD1 and gadD3 
even more by sodium maleate $(\mathrm{P}<0.05$, paired t-test, Fig. $6 \mathrm{~B})$. The above suggest that $L$. monocytogenes tries to counteract the inhibitory effects of fumarate and maleate on GAD activity by increasing gadD2 transcription and the final result of these opposing actions in the case of fumarate is increased $\mathrm{GAD}_{\mathrm{e}}$ system output which however, does not enhance acid resistance. However, in the case of maleate, higher increase in gadD2 transcription is unable to increase $\mathrm{GAD}_{\mathrm{e}}$ system output (main difference with fumarate) but similarly to fumarate, the acid resistance is reduced.

The explanation for the antilisterial effects of fumarate might lie in the effects on the $\mathrm{GAD}_{\mathrm{i}}$ system (Feehily and Karatzas, 2013), or other possible effects on other acid resistance systems or on cell metabolism that in its turn could affect acid resistance. For example, fumarate is highly antimicrobial against organisms such as Salmonella (Kondo et al., 2006) that lack GAD system suggesting these additional effects (Park et al., 1996). To assess this, we first looked at the aminoacid profile in presence or absence of fumarate and the only difference found was the increased $\mathrm{GABA}_{\mathrm{e}}$ levels in presence of fumarate confirming the GABase results. This suggests that other aminoacid decarboxylase systems are possibly not affected and the above effects of fumarate are on $\mathrm{GAD}_{\mathrm{i}}$ system or possibly another non-amino acid decarboxylase system.

464 Furthermore, the ability of sodium fumarate to act on cells in a biofilm was examined. It has previously been shown that maleic acid can act on biofilms of L. monocytogenes (Paudyal et al., 2018) and E. faecalis (Ferrer-Luque et al., 2010). Due to these properties it has been 467 suggested that maleic acid could be an effective alternative to the more toxic EDTA commonly used to remove biofilms from the oral cavity and dental equipment (Ballal et al., 2009; Ferrer469 Luque et al., 2010). However, fumarate has no toxicity and therefore further work could investigate other potential applications. Our results showed the striking ability of sodium

471 fumarate $(25 \mathrm{mM})$ to eliminate cells of three different strains of L. monocytogenes in a biofilm, 472 which was significantly higher than that of hypochlorite and a commonly used organic acid 
473 disinfectant AM at pH 2.4 (Fig. 7). Furthermore, the addition of fumarate together with the AM

474 disinfectant increased significantly the ability of the disinfectant to eliminate cells in biofilm.

475 Our results also show that the more acid resistant strain 10403S survived the treatments better

476 than the other two acid sensitive strains (EGD-e and LO28), underpinning the important role

477 of acid resistance and GAD system in survival in a biofilm. Furthermore, we also found that

478 LO28 was highly sensitive to chlorine. This coincides with previous reports suggesting a high

479 variation in resistance to chlorine-based sanitisers among different strains (Brackett, 1987;

480 Jacquet and Reynaud, 1994) and that mixed culture strains of L. monocytogenes are better able

481 to resist chlorine treatments (Vaid et al., 2010). Our results suggest that fumarate has a great

482 potential for removal of biofilms of L. monocytogenes while it is also nontoxic.

484 Conclusions

485 Overall, we investigated the effect of fumarate on L. monocytogenes under acidic conditions 486 showing that although it is a $\mathrm{GAD}_{\mathrm{e}}$ inhibitor, the bacterium is able to counteract this with 487 increased transcription, being able to increase its overall $\mathrm{GAD}_{\mathrm{e}}$ output, which however does 488 not translate into increased acid resistance. We also show that there is a difference between 489 fumarate and maleate as although the first increases GAD output, the latter reduces it, but both 490 significantly enhance death under acidic conditions. The antimicrobial activity of fumarate 491 might be related to reduced $\mathrm{GAD}_{\mathrm{i}}$ or other systems. Further work is required to elucidate the 492 full extent of the antimicrobial activity of fumarate on L. monocytogenes and other organisms. 493 Such work will allow us to successfully eliminate this pathogen in food and food preparation 494 environments but also explain its behaviour in environments where fumarate is present. 495

\section{Acknowledgements}


The authors would like to thank all colleagues at the Microbiology research team and the experiments has been invaluable. The work was supported by a Biotechnology and Biological

Sciences Research Council doctoral training partnership (BBSRC-DTP Grant number RE04907), awarded to Dr. K.A.G. Karatzas and funds from the University of Reading and by AgriCoat NatureSeal Ltd.

\section{References}

Abrams, F., Wan-Lin, S., Wiedmann, M., Boor, K.J., Coote, P.J., Botting, C.H., Karatzas, K.A.G., O'Byrne, C.P., (2008). Proteomic analysis of a Listeria monocytogenes mutant lacking sigma $\mathrm{B}\left(\sigma^{\mathrm{B}}\right)$ identify new components of the $\sigma^{\mathrm{B}}$ regulon and highlight a role for sigma B in the utilization of glycerol. Appl. Environ. Microbiol. 74, 594604.

Arnold, C.N., McElhanon, J., Lee, A., Leonhart, R., Siegele, D.A., (2001). Global analysis of Escherichia coli gene expression during the acetate-induced acid tolerance response. J. Bacteriol. 183.

Ballal, N.V., Kandian, S., Mala, K., Bhat, K.S., Acharya, S., (2009). Comparison of the efficacy of maleic acid and ethylenediaminetetraacetic acid in smear layer removal from instrumented human root canal: a scanning electron microscopic study. $J$. Endod. 35, 1573-1576.

Boura, M., Keating, C., Royet, K., Paudyal, R., O'Donoghue, B., O'Byrne, C.P., Karatzas, K.A., (2016). Loss of SigB in Listeria monocytogenes strains EGD-e and 10403S confers hyperresistance to hydrogen peroxide in stationary phase under aerobic conditions. Appl. Environ. Microbiol. 82, 4584-4591.

Brackett, R., (1987). Antimicrobial effect of chlorine on Listeria monocytogenes. J. Food Prot. 50, 999-1003.

Chikthimmah, N., Laborde, L., Beelman, R., (2003). Critical factors affecting the destruction of Escherichia coli O157: $\mathrm{H} 7$ in apple cider treated with fumaric acid and sodium benzoate. J. Food Sci. 68, 1438-1442. 
Comes, J.E., Beelman, R.B., (2002). Addition of fumaric acid and sodium benzoate as an alternative method to achieve a 5-log reduction of Escherichia coli O157: H7 populations in apple cider. J. Food Prot. 65, 476-483.

Cotter, P.D., Gahan, C.G., Hill, C., (2001). A glutamate decarboxylase system protects Listeria monocytogenes in gastric fluid. Mol. Microbiol. 40, 465-475.

Cotter, P.D., Hill, C., (2003). Surviving the acid test: Responses of Gram-positive bacteria to low pH. Microbiol. Mol. Biol. Rev. 67, 429-429.

Cotter, P.D., Ryan, S., Gahan, C.G., Hill, C., (2005). Presence of GadD1 glutamate decarboxylase in selected Listeria monocytogenes strains is associated with an ability to grow at low pH. Appl. Environ. Microbiol. 71, 2832-2839.

Davis, M.J., Coote, P.J., O'Byrne, C.P., (1996). Acid tolerance in Listeria monocytogenes: the adaptive acid tolerance response (ATR) and growth-phase-dependent acid resistance. Microbiol. 142 ( Pt 10), 2975-2982.

Elmore, J.S., Koutsidis, G., Dodson, A.T., Mottram, D.S., Wedzicha, B.L., (2005). Measurement of acrylamide and its precursors in potato, wheat, and rye model systems. J. Agric. Food Chem. 53, 1286-1293.

Feehily, C., Finnerty, A., Casey, P.G., Hill, C., Cormac, G.M.G., O'Byrne, C.P., Karatzas, K.A.G., (2014). Divergent evolution of the activity and regulation of the glutamate decarboxylase systems in Listeria monocytogenes EGD-e and 10403S: Roles in virulence and acid tolerance:. PLOS 9 (11), e112649.

Feehily, C., Karatzas, K.A.G., (2013). Role of glutamate metabolism in bacterial responses towards acid and other stresses. J. Appl. Microbiol. 114, 11-24.

Feehily,C., O'Byrne, CP,. Karatzas, KAG,. (2013) Functional $\gamma$-Aminobutyrate shunt in Lisitria Monocytognese: role in acid tolerance and succinatebiosynthesis. Appl. Environ. Microbiol.(79), 74-80.

Ferrer-Luque, C.M., Arias-Moliz, M.T., Gonzalez-Rodriguez, M.P., Baca, P., (2010). Antimicrobial activity of maleic acid and combinations of cetrimide with chelating agents against Enterococcus faecalis biofilm. J. Endod. 36, 1673-1675.

Fonda, M.L., (1972). Glutamate decarboxylase. Substrate specificity and inhibition by carboxylic acids. Biochemistry (Easton) 11, 1304-1309.

Food Standards Agency, (2011). Foodborne disease strategy 2010-15, in: agency, F.s. (Ed.).

Foster, J.W., (2004). Escherichia coli acid resistance: tales of an amateur acidophile. Nat. Rev. Microbiol. 2, 898-907.

Grobelny, J., (1995). N.m.r. study of maleate (cis) -fumarate (trans) isomerism in unsaturated polyesters and related compounds. Polymer. 36, 4215-4222. 
Heersink J., (2003). Basic biofilm analytical methods. In: Hamilton, M., Heersink, J., Buckingham-Meyer, K. Goeres, D. (Ed 4th.) The biofilms laboratory: Step-by-step protocols for experimental design, analysis, and data interpretation. Bozeman: Cytergy Publishing, pp. 16-23.

Jacquet, C., Reynaud, A., (1994). Differences in the sensitivity to eight disinfectants of Listeria monocytogenes strains as related to their origin (short note). Int. J. Food Microbiol. 22, 79-83.

Karatzas, K.-A.G., Suur, L., O'Byrne, C.P., (2012). Characterization of the intracellular glutamate decarboxylase system: Analysis of its function, transcription, and role in the acid resistance of various strains of Listeria monocytogenes. Appl. Environ. Microbiol. 78, 3571-3579.

Karatzas, K.A., Brennan, O., Heavin, S., Morrissey, J., O'Byrne, C.P., (2010). Intracellular accumulation of high levels of gamma-aminobutyrate by Listeria monocytogenes $10403 \mathrm{~S}$ in response to low $\mathrm{pH}$ : uncoupling of gamma-aminobutyrate synthesis from efflux in a chemically defined medium. Appl. Environ. Microbiol. 76, 3529-3537.

Kim, Y.J., Kim, M.H., Song, K.B., (2009). Efficacy of aqueous chlorine dioxide and fumaric acid for inactivating pre-existing microorganisms and Escherichia coli O157:H7, Salmonella Typhimurium, and Listeria monocytogenes on broccoli sprouts. Food Control. 20, 1002-1005.

Kondo, N., Murata, M., Isshiki, K., (2006). Efficiency of sodium hypochlorite, fumaric acid, and mild heat in killing native microflora and Escherichia coli O157: H7, Salmonella Typhimurium DT104, and Staphylococcus aureus attached to fresh-cut lettuce. J. Food Prot, 69, 323-329.

Lambert, R., Stratford, M., (1999). Weak-acid preservatives: modelling microbial inhibition and response. J. Appl. Microbiol. 86, 157-164.

Lee, B.H., (2014). Fundamentals of Food Biotechnology $2^{\text {nd }}$ edn. John Wiley \& Sons, Incorporated, New York, NY, 1413 pp.

Liu, S., Graham, J.E., Bigelow, L., Morse, P.D., Wilkinson, B.J., (2002). Identification of Listeria monocytogenes genes expressed in response to growth at low temperature. Appl. Environ. Microbiol. 68, 1697-1705.

Lohbeck, K., Haferkorn, H., Fuhrmann, W., Fedtke, N., (2000). Maleic and fumaric acids. Ullmann's encyclopedia of industrial chemistry Wiley-VCH, Weinheim.

Miller, L.G., Kaspar, C.W., (1994). Escherichia coli O157: H7 acid tolerance and survival in apple cider. J. Food Prot. 57, 460-464.

O'Byrne, C.P., Feehily, C., Ham, R., Karatzas, K.A.G., (2011). A modified rapid enzymatic microtiter plate assay for the quantification of intracellular $\gamma$-aminobutyric acid and succinate semialdehyde in bacterial cells. J. Microbiol. Meth. 84, 137-139. 
O'Driscoll, B., Gahan, C.G., Hill, C., (1996). Adaptive acid tolerance response in Listeria monocytogenes: isolation of an acid-tolerant mutant which demonstrates increased virulence. Appl. Environ. Microbiol. 62, 1693-1698.

Ohno, M., Okunuki, K., (1962). Studies on the inhibition of glutamic acid decarboxylase of squash. J. Biochem. 51, 313-316.

Okuyama, T., Maskill, H., (2013). Organic chemistry: a mechanistic approach. Oxford University Press.

Park, Y.K., Bearson, B., Bang, S.H., Bang, I.S., Foster, J.W., (1996). Internal pH crisis, lysine decarboxylase and the acid tolerance response of Salmonella Typhimurium. Mol Microbiol. 20, 605-611.

Paudyal, R., Barnes, R.H., Karatzas, K.A.G., (2018). A novel approach in acidic disinfection through inhibition of acid resistance mechanisms; Maleic acid-mediated inhibition of glutamate decarboxylase activity enhances acid sensitivity of Listeria monocytogenes. Food Microbiol. 69, 96-104.

Pérez-Díaz, I., McFeeters, R., (2010). Preservation of acidified cucumbers with a natural preservative combination of fumaric acid and allyl isothiocyanate that target lactic acid bacteria and yeasts. J. Food Sci. 75 (4). doi: 10.1111/j.1750-3841.2010.01587.x.

Podolak, R., Zayas, J., Kastner, C., Fung, D., (1996). Inhibition of Listeria monocytogenes and Escherichia coli O157: $\mathrm{H} 7$ on beef by application of organic acids. J. Food Prot. 59, 370-373.

Posfay-Barbe, K.M., Wald, E.R., (2009). Listeriosis. Semin. Fetal Neonatal Med. 14, 228 233.

Ramírez, M.D.F., Smid, E.J., Abee, T., Groot, M.N.N., (2015). Characterisation of biofilms formed by Lactobacillus plantarum WCFS1 and food spoilage isolates. Int. J. Food Microbiol. 207, 23-29.

Ricke, S., (2003). Perspectives on the use of organic acids and short chain fatty acids as antimicrobials. Poult. Sci. 82, 632-639.

Roa Engel, C.A., ter Horst, J.H., Pieterse, M., van der Wielen, L.A., Straathof, A.J., (2013). Solubility of fumaric acid and its monosodium salt. Ind. Eng. Chem. Res. 52, 94549460.

Saltmarsh, M., Barlow, S., Richardson, V., Robin, A.-L., Jukes, D.J., (2013). Essential guide to food additives. Royal Society of Chemistry, Cambridge.

Szalka, M., Rokaszewski, E., Kaczmarski, K., (2013). Kinetics of hydrolysis of bisoprolol hemifumarate in aqueous acidic solutions. Int. J. Chem. Kinet. 45, 744-754.

Vaid, R., Linton, R.H., Morgan, M.T., (2010). Comparison of inactivation of Listeria monocytogenes within a biofilm matrix using chlorine dioxide gas, aqueous chlorine dioxide and sodium hypochlorite treatments. Food Microbiol. 27, 979-984. 
685
Wemmenhove, E., van Valenberg, H.J.F., Zwietering, M.H., van Hooijdonk, T.C.M., WellsBennik, M.H.J., (2016). Minimal inhibitory concentrations of undissociated lactic, acetic, citric and propionic acid for Listeria monocytogenes under conditions relevant to cheese. Food Microbiol. 58, 63-67.

Zhou, Y., Du, J., Tsao, G., (2002). Comparison of fumaric acid production by Rhizopus oryzae using different neutralizing agents. Bioprocess Biosyst. Eng. 25, 179-181.

\section{Legends to the figures}

Fig. 1. Survival of L. monocytogenes (A) 10403S WT in the presence (black circles) and absence (black triangles) of $8.6 \mathrm{mM}$ sodium fumarate adjusted to $\mathrm{pH} 3$ using $1 \mathrm{M} \mathrm{HCl}$ (B) EGD-e WT and (C) LO28 WT in the presence (black circles) and absence (black triangles) of $4.3 \mathrm{mM}$ sodium fumarate at $\mathrm{pH} 3.3 \mathrm{using} 1 \mathrm{M} \mathrm{HCl}$. Asterisks represent statistically significant result ( $\mathrm{P}<0.05$ paired student $\mathrm{T}$-test) while D.L denotes detection limit of the experimental setup.

Fig. 2. Survival of L. monocytogenes and its GAD mutants in the (A) 10403S background in the presence and absence of $8.6 \mathrm{mM}$ of sodium fumarate adjusted to $\mathrm{pH} 3$ for $60 \mathrm{~min}$, in the (B) LO28 and (C) EGD-e background in the presence and absence of $4.3 \mathrm{mM}$ of sodium fumarate adjusted to $\mathrm{pH} 3.3$ for $15 \mathrm{~min}$. Adjustment of $\mathrm{pH}$ was done using $1 \mathrm{M} \mathrm{HCl}$. Asterisks represent statistically significant result as assessed with paired student $\mathrm{T}$-test $(\mathrm{P}<0.05)$ and M.L. denotes the maximum log reduction could be recorded with the current protocol.

Fig. $3 \mathrm{GABA}_{\mathrm{e}}$ levels of overnight cultures grown to stationary phase $\left(\sim 18 \mathrm{~h}\right.$ at $\left.37^{\circ} \mathrm{C}\right)$ with shaking in the presence or absence of $10 \mathrm{mM}$ sodium fumarate (SF) for (A) L. monocytogenes $10403 \mathrm{WT}$ at $\mathrm{pH} 4.2$, (B) L. monocytogenes LO28 WT at pH 4.2 and (C) E. coli K-12 WT at $\mathrm{pH}$ 4. $\mathrm{pH}$ was adjusted with the addition of $1 \mathrm{M} \mathrm{HCl}$. Asterisk represents statistically significant result. $\mathrm{P}<0.05$ paired student T-test. 
Fig. 4. GAD activity in cell lysates of L. monocytogenes 10403S WT cells grown overnight until stationary phase $(\sim 18 \mathrm{~h})$ at $37^{\circ} \mathrm{C}$ with agitation $(150 \mathrm{rpm})$ in the presence or absence of 20 $\mathrm{mM}$ sodium fumarate at $\mathrm{pH}$ 4.2. Lysates were prepared and then levels of GAD activity were assessed using GC-MS. Asterisk represents statistically significant result. $\mathrm{P}<0.05$ paired student T-test.

Fig. 5 GAD activity in cell lysates of L. monocytogenes 10403S WT, EGD-e WT and LO28 WT cells grown overnight until stationary phase $(\sim 18 \mathrm{~h})$ at $37^{\circ} \mathrm{C}$ with agitation $(150 \mathrm{rpm})$. Lysates were produced and then levels of GAD activity were assessed using the GABase enzymatic assay. Asterisks represents statistically significant result. $\mathrm{P}<0.05$ paired student $\mathrm{T}$ test.

Fig. 6 Expression of (A) gadD1 (B) gadD2 and (C) gadD3 gene in L. monocytogenes 10403S WT in the absence or presence of $10 \mathrm{mM}$ sodium fumarate or $10 \mathrm{mM}$ maleic acid. Relative expression of each gene was calculated by comparing expression relative to 16S rRNA gene in each strain. Numbers above the bars represent fold difference in relative expression compared to control. Markers represent an average of triplicate measurements and error bars represent standard deviations.Asterisks * denote statistical significant difference compared to the control ( $\mathrm{P}<0.05$ paired student T-test).

Fig. 7 Survival of cells in biofilms of L. monocytogenes (A) 10403S WT (B) L. EGD-e WT and (C) LO28 WT following no treatment (water) or treatment with an acidic disinfectant (AM), $\mathrm{AM}$ together with $25 \mathrm{mM}$ sodium fumarate (SF), $\mathrm{HCl}$ and $\mathrm{HCl}$ together with $25 \mathrm{mM}$ SF. All treatments were at $\mathrm{pH}$ 2.4. Asterisks represent statistically significant difference between no treatment and a treatment $(\mathrm{P}<0.05$; paired student T-test $)$. 
756

757

758

759

760

$761 \quad$ Tables

762

Table 1. list of strains used in these experiments

\begin{tabular}{|c|c|c|}
\hline Strains & Relevant properties & Source \\
\hline L. monocytogenes $10403 \mathrm{~S}$ & Serotype $1 / 2 \mathrm{a}, \mathrm{WT}$ & Karatzas et al., 2010 \\
\hline L. monocytogenes $10403 \mathrm{~S} \Delta$ gadD1 & 10403S with gadD1 deleted & Karatzas et al., 2010 \\
\hline L. monocytogenes $10403 \mathrm{~S} \Delta$ gadD2 & 10403S with gadD2 deleted & Karatzas et al., 2010 \\
\hline L. monocytogenes $10403 \mathrm{~S} \Delta$ gadD3 & 10403S with gadD3 deleted & Karatzas et al., 2010 \\
\hline L. monocytogenes EGD-e & Serotype 1/2a, WT & Feehily et al., 2013 \\
\hline L. monocytogenes EGD-e $\Delta$ gadDl & EGD-e with gadDI deleted & Feehily et al., 2013 \\
\hline L. monocytogenes EGD-e $\Delta$ gadD2 & EGD-e with gadD2 deleted & Feehily et al., 2013 \\
\hline L. monocytogenes EGD-e $\Delta$ gadD3 & EGD-e with gadD3 deleted & Feehily et al., 2013 \\
\hline L. monocytogenes LO28 & Serotype $1 / 2 \mathrm{c}, \mathrm{WT}$ & Cotter et al., 2001 \\
\hline L. monocytogenes LO28 $\Delta$ gadD1 & LO28 with gadD1 deleted & Cotter et al., 2001 \\
\hline L. monocytogenes $\mathrm{LO} 28 \Delta$ gadD2 & LO28 with gadD2 deleted & Cotter et al., 2001 \\
\hline L. monocytogenes LO28 $\Delta$ gadD1/2 & LO28 with $\mathrm{gad}$ D1/2 deleted & Cotter et al., 2001 \\
\hline
\end{tabular}


765

766

769

770

771

772

773 Table 2. Percentage of undissociated acids at pH 3.

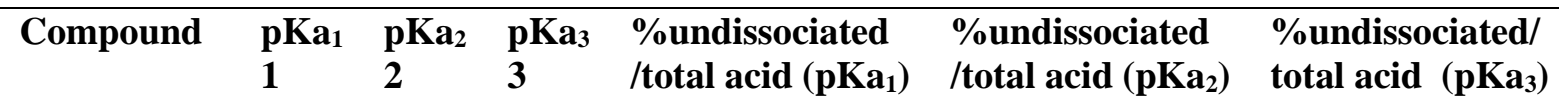

\begin{tabular}{|c|c|c|c|c|c|c|}
\hline Maleic acid & 1.9 & 6.07 & & 7.35 & 99.91 & \\
\hline $\begin{array}{l}\text { Fumaric } \\
\text { acid }\end{array}$ & 3.03 & 4.44 & & 51.72 & 96.49 & \\
\hline $\begin{array}{l}\text { Sodium } \\
\text { fumarate }\end{array}$ & 3.55 & & & 78.01 & & \\
\hline Pimlic acid & 4.71 & 5.58 & & 98.08 & 99.73 & \\
\hline Valeric acid & 4.82 & & & 98.50 & & \\
\hline Adipic acid & 4.43 & 5.41 & & 96.41 & 99.61 & \\
\hline $\begin{array}{l}\text { Glutaric } \\
\text { acid }\end{array}$ & 4.34 & 5.22 & & 95.62 & 99.40 & \\
\hline Malic acid & 3.4 & 5.44 & & 71.52 & 99.63 & \\
\hline Citric acid & 3.13 & 4.76 & 6.39 & 57.42 & 98.29 & 99.95 \\
\hline $\begin{array}{l}\text { Tartaric } \\
\text { acid }\end{array}$ & 2.98 & 4.34 & & 48.84 & 95.62 & \\
\hline $\begin{array}{l}\text { Oxaloacetic } \\
\text { acid }\end{array}$ & 2.22 & 3.89 & & 14.23 & 88.58 & \\
\hline
\end{tabular}




\begin{tabular}{|c|c|c|}
\hline $\begin{array}{l}\text { Alpha } \\
\text { ketoglutaric } \\
\text { acid }\end{array}$ & 3.08 & 54.59 \\
\hline Valeric acid & 4.82 & 98.50 \\
\hline $\begin{array}{l}\text { Levulinic } \\
\text { acid }\end{array}$ & 4.59 & 97.49 \\
\hline
\end{tabular}

Table 3. MICs of compounds tested

\begin{tabular}{cc}
\hline Potential inhibitor & MIC \\
\hline Maleic acid & $34.4 \mathrm{mM}$ \\
Fumaric acid & $34.4 \mathrm{mM}$ \\
Sodium fumarate & $\begin{array}{c}\text { Above solubility } \\
\text { threshold } 0.22 \mathrm{mg} / \mathrm{ml}\end{array}$ \\
& \\
Glutaric acid & $30 \mathrm{mM}$ \\
Pimelic acid & $24.9 \mathrm{mM}$ \\
Adipic acid & $27.3 \mathrm{mM}$ \\
Malic acid & $14.9 \mathrm{mM}$ \\
Citric acid & $20.8 \mathrm{mM}$ \\
Tartaric acid & $26.6 \mathrm{mM}$ \\
Oxaloacetic acid & $60.5 \mathrm{mM}$ \\
$\alpha$-Ketoglutaric acid & $27.3 \mathrm{mM}$ \\
Valeric acid & $13 \mathrm{mM}$ \\
Levulinic acid & $60 \mathrm{mM}$ \\
\hline
\end{tabular}

